\title{
Thermal Stability of Nanocrystalline Ni
}

\author{
A.V. Korznikov ${ }^{a}$, Z. PAKIEŁA ${ }^{b}$ AND K.J. KurZYdŁOWski ${ }^{b}$ \\ ${ }^{a}$ Institute of Problems of Metal Superplasticity, Russian Academy of Sciences \\ Khalturina 39, Ufa, 450001 Bashkortostan, Russia \\ ${ }^{b}$ Faculty of Materials Science and Engineering, Warsaw University of Technology \\ Wołoska 141, 02-507 Warsaw, Poland
}

\begin{abstract}
Ultrafine-grained structure with grain size of about $100 \mathrm{~nm}$ was obtained in nickel by deformation under a pressure of $7 \mathrm{GPa}$ in Bridgman anvils. The structure evolution in ultrafine-grained nickel was studied by residual resistance, transmission electron microscopy, X-ray diffraction, and microhardness measurements. It was established that the evolution of the structure upon heating of ultrafine-grained nickel may be divided into three stages. Stage A corresponds to temperatures below $453 \mathrm{~K}$ and is characterised by an insignificant decrease in the resistivity and microhardness. At this stage, a decrease in the internal stresses is not accompanied by grain growth. Stage B, corresponding to the temperatures range of $453-513 \mathrm{~K}$, is characterised by an abrupt decrease in the resistivity and hardness, disappearance of the internal stresses, and by an intense grain growth. Stage $\mathrm{C}$ (above $523 \mathrm{~K}$ ) corresponds to an insignificant increase in the resistivity and further decrease in the hardness.
\end{abstract}

PACS numbers: 62.25.+g, 73.63.Bd

\section{Introduction}

The metals and alloys with ultrafine-grained structures are of interest due to their beneficial combination of properties, such as high low-temperature strength and high-temperature plasticity [1,2]. In order to ensure a wider application of these alloys, the relationship between their structure, properties, and thermal stability should be studied in detail. The nanocrystalline materials prepared by different methods such as crystallisation from the amorphous state, consolidation of powder, intense plastic deformation, etc., have been studied in many projects [1-3]. However, there is no consensus on which structural factors are of the key importance. The dominant role of grain boundaries in the formation of physical and mechanical properties of nanocrystalline materials was shown in [1-3] and 
the present paper focus is on their role in nanocrystals obtained via severe plastic deformation (SPD).

This work aims to study the nanocrystalline structure of SPD nickel and the evolution and stability of its microstructure under thermal treatment.

\section{Experimental}

In order to produce a nanocrystalline structure, $99.95 \%$-pure nickel obtained by vacuum melting was deformed by torsion at room temperature under a pressure of $7 \mathrm{GPa}$ to a true strain $e=7$. The specimens were annealed at $300-700 \mathrm{~K}$ for 30 min in a vacuum of $10^{-4} \mathrm{~Pa}$.

The microstructure was examined by the conventional methods of transmission electron microscopy in a JEM-2000EX instrument and by X-ray diffraction using the technique described in $[4,5]$. The latter method was used to estimate the grain size (coherent domains) and the magnitude of elastic deformations (internal stresses).

Microhardness was measured in a Vickers tester at a load of $0.2 \mathrm{~kg}$ and an indentation time of $10 \mathrm{~s}$. Resistivity was measured by the four-probe technique at $4.2 \mathrm{~K}$ employing the concept of a geometrical shape factor as in [6]. The measurement error was within $5 \times 10^{-9} \Omega \mathrm{cm}$.

\section{Results}

As was shown by the electron-microscopic examination, nickel subjected to heavy plastic deformation has a nonuniform fine-grained structure. Regions near grain boundaries often exhibit a high dislocation density. The average grain size, estimated from dark-field images is $100 \mathrm{~nm}$. These data are consistent with small-area electron-diffraction patterns $\left(0.5 \mu \mathrm{m}^{2}\right)$ that contain a high number of spots forming a diffraction ring. This indicates the presence of very fine, strongly misoriented grains. The typical microstructures and the electron-diffraction patterns are shown in Fig. 1. The average grain size estimated from the X-ray diffraction data is in good agreement with that obtained from electron-microscopic data (Fig. 2d).

It was also found that the material in this state is characterised by a high resistivity $\left(\rho_{4.2}=0.26 \mu \Omega \mathrm{cm}\right)$, a high elastic deformation $(\Delta a / a=0.076 \%$, where $a$ is the lattice parameter) and the microhardness HV $=2850 \mathrm{MPa}$ (Fig. 2). These values indicate the presence of a high density of lattice defects and a high level of internal stresses.

The microstructure of the specimens changes with increasing annealing temperature in the following manner. At low temperatures, the dislocation density somewhat decreases and the grain growth starts at approximately $400 \mathrm{~K}$ (Fig. 2d). Higher annealing temperatures cause a recrystallization accompanied by decreasing volume fraction of a nanocrystalline structure (Fig. 3a). The recrystallized 
a)
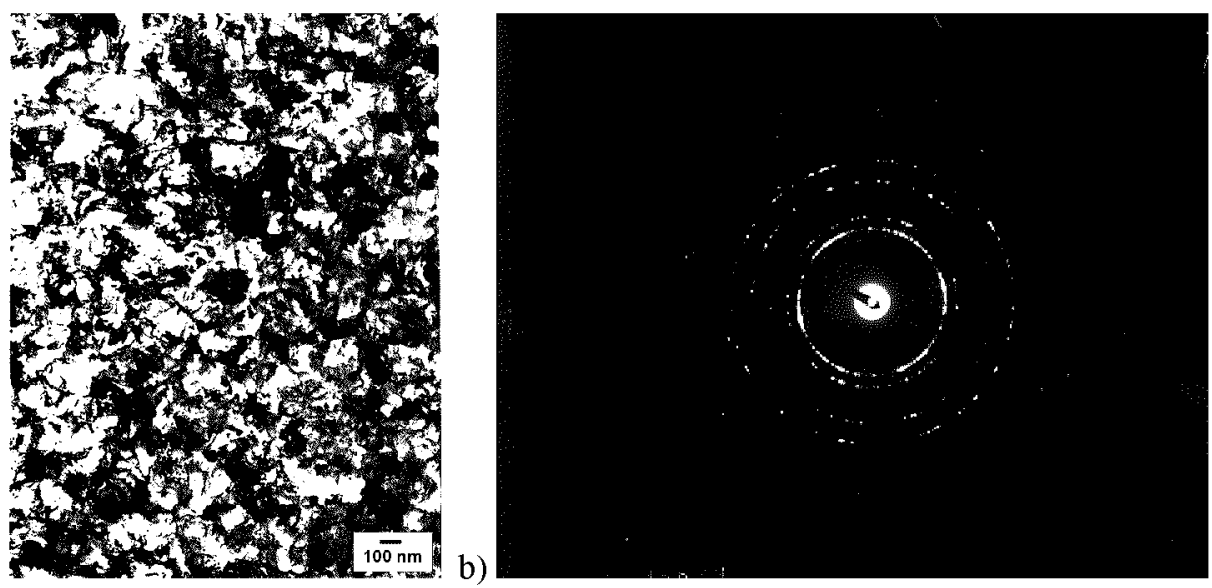

Fig. 1. Microstructure of nanocrystalline nickel after heavy plastic deformation: (a) bright-field and (b) electron-diffraction pattern taken from a $0.5 \mu \mathrm{m}^{2}$ area.

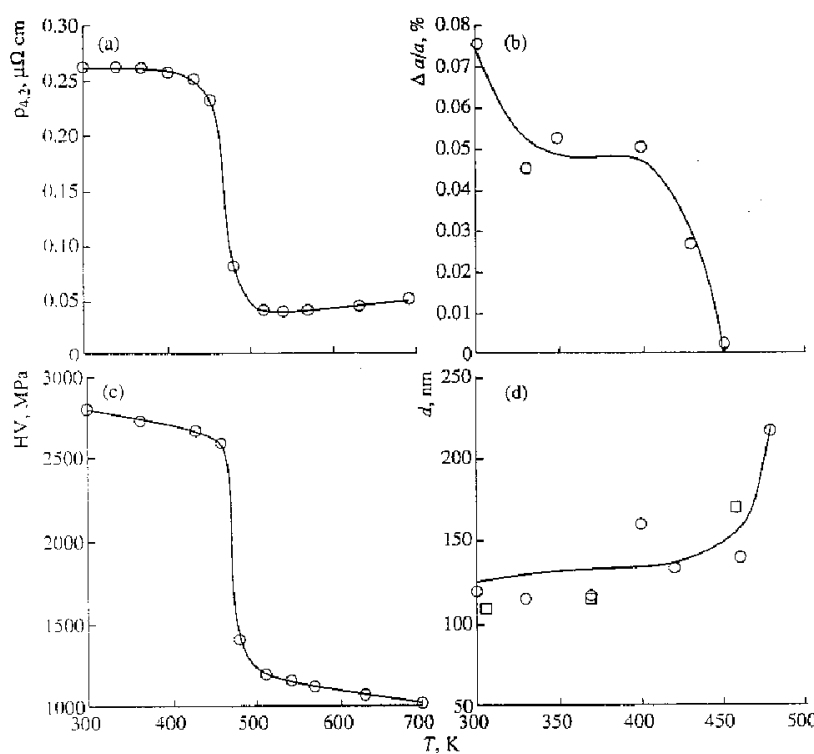

Fig. 2. Effect of annealing temperature $(0.5 \mathrm{~h})$ on the characteristics of nickel: (a) resistivity $\rho_{4,2}$ at $4.2 \mathrm{~K}$, (b) internal elastic stresses $\Delta a / a$ : (c) microhardness $\mathrm{HV}$, and (d) grain size $d$ : (o) electron-microscopic data and ( $\square$ ) X-ray diffraction data (the $\mathrm{X}$-ray diffraction data on $\Delta a / a$ and $d$ are obtained from the broadening of the (220) line).

grains contain virtually no dislocations. An intense grain coarsening is observed at temperatures above $450 \mathrm{~K}$ : the grain sizes in the specimens annealed at 573 
and $673 \mathrm{~K}$ are 1.8 and $3.1 \mu \mathrm{m}$, respectively. At this stage the structure looks like a fully recrystallized one, and virtually no dislocations are observed in the bulk of grains (Fig. 3b).

a)

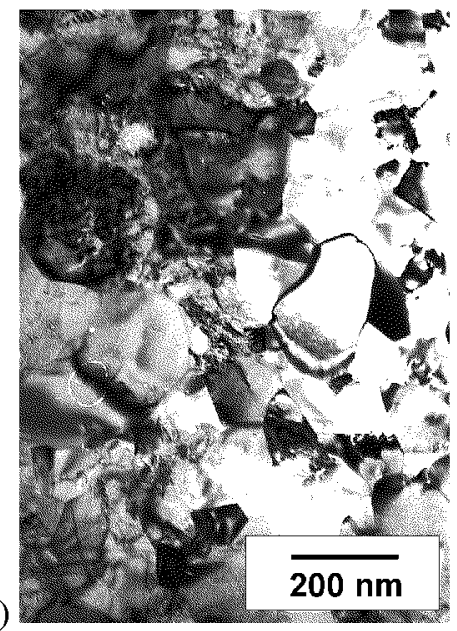

b)

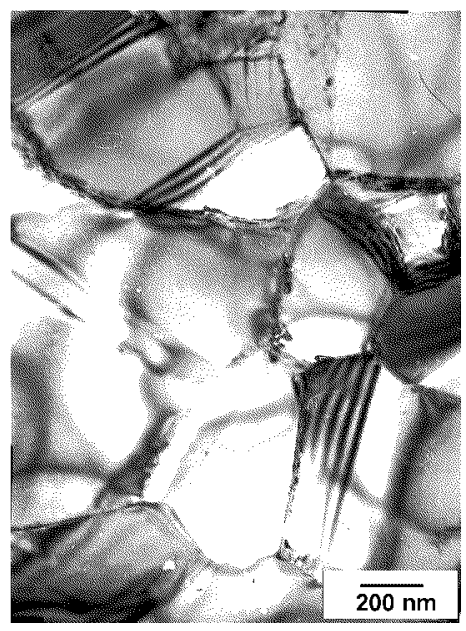

Fig. 3. Bright-field images of nickel specimens annealed for $30 \mathrm{~min}$ at (a) 473 and (b) $673 \mathrm{~K}$.

Concurrently with the above changes in material structure with increasing annealing temperature, an abrupt decrease in the level of internal stresses is observed at 300-350 K. Subsequently stress stabilisation occurs at temperatures up to $400 \mathrm{~K}$, and the stress relaxation observed at higher temperatures causes the complete disappearance of stresses at $450 \mathrm{~K}$ (Fig. 2b).

The variation in the resistivity and microhardness as a function of the annealing temperature is shown in Figs. $2 a$ and c. It is seen that these characteristics decrease abruptly at temperatures from 450 to $500-525 \mathrm{~K}$. At the same time, at lower $(300-150 \mathrm{~K})$ and higher $(525-700 \mathrm{~K})$ annealing temperatures, the resistivity virtually does not change, and the microhardness decreases only slightly.

\section{Discussion}

Based on the results reported here one may recognise three stages of the evolution of structure and properties with increasing temperature. These stages correspond to temperature ranges of $293-450 \mathrm{~K}, 450-525 \mathrm{~K}$, and $525-700 \mathrm{~K}$.

As far as the variation in internal stresses is concerned it should be noted that an intense plastic deformation causes a highly nonequilibrium state of material $[3,5,7,8]$. Thus the nanocrystalline materials produced by very large deformations are highly nonequilibrium [9]. The annealing of such materials causes structural changes that begin in the regions with the highest defect density. 
In our case, the redistribution and possible annihilation of dislocations and defects in grain-boundary regions are likely to take place during the annealing inside the nanograins. These processes, promoting the relaxation of internal stresses, intensify with increasing annealing temperature. It is likely that the annealing time of $30 \mathrm{~min}$ at $350 \mathrm{~K}$ is sufficient for this rearrangement, and, as the annealing temperature increases somewhat further, virtually no changes occur.

However, annealing at $400 \mathrm{~K}$ stimulates grain growth and grain-boundary migration. The moving boundaries adsorb ("sweep") dislocations encountered in their way and leave dislocation-free regions behind themselves. At the same time they become closer to equilibrium, and contain fewer grain-boundary defects. All these processes decrease internal stresses. At higher temperatures, an intense recrystallization takes place and leads to effective stress relaxation, which is already completed at about $450 \mathrm{~K}$. A further increase in temperature is accompanied by grain growth in the absence of internal stresses. This interpretation is consistent with the above structure observations and with the model of the formation and relaxation of internal stresses in ultrafine-grained materials [10]. Let us note also that recently a high density of grain-boundary dislocations and related stresses were observed in ultrafine-grained nickel produced by heavy plastic deformation [11].

As follows from the foregoing, it is reasonable to attribute the continuous decrease in microhardness with increasing annealing temperature at the first stage to the observed rearrangement of the defect structure at grain boundaries and in the adjacent regions, i.e., to decreasing level of internal stresses. In this case, the virtually constant resistivity implies that the rearrangement in the structure increases its equilibrium, but does not substantially affect the defect density.

By contrast, the abruptly decreasing microhardness and resistivity at the second stage are associated with an abruptly decreasing dislocation density and number of grain-boundary defects (including grain-boundary dislocations) in the process of recrystallization. In fact, the change in the electrical resistivity, caused by scattering of electrons by dislocations, is $\Delta \rho \leq 10^{-13}(d / b) \rho_{\mathrm{g}} \mu \Omega \mathrm{cm}$, where $\rho_{\mathrm{g}}$ is the dislocation density, $d$ is the dislocation splitting, and $b$ is the Burgers vector [12]. In our case, the grain size in the nanocrystalline nickel is $100 \mathrm{~nm}$, the resistivity changes by $0.22 \mu \Omega \mathrm{cm}$ (Fig. 2a), and $d$ for nickel is equal to $4 b$ [12]. Then, taking the width of the grain-boundary region to be equal to 0.1 of the grain radius, we obtain $\rho_{\mathrm{g}} \geq 3 \times 10^{12} \mathrm{~cm}^{-2}$. According to the theory [13], the separate dislocations cannot be resolved in the regions with such a high dislocation density. Their images merge, resulting in the appearance of dark areas (in bright-field images) in grain-boundary regions. Such regions have indeed been observed here (Fig. 1). A smooth decrease in microhardness with increasing annealing temperature at the third stage can be reasonably associated with the grain growth. A decrease in the resistivity after annealing at this stage may be caused by the appearance of quench defects (e.g., vacancies) upon cooling of the speci- 
mens from the annealing temperatures. Then the effect should be enhanced with increasing annealing temperature, as it is actually observed in our experiments. Note that the above interpretation requires that natural "scatter" in the occurrence of the annealing-induced processes in different regions of the specimen (at a certain temperature) should be taken into account. Therefore, the temperature ranges corresponding to the annealing stages may be shifted, and the stages may overlap in various regions of specimens.

\section{Conclusions}

(1) The nanocrystalline structure obtained in nickel by severe plastic deformation, as well as the characteristics of nickel in this state, such as the internal elastic stresses, microhardness, and resistivity, after deformation and subsequent annealing can be explained in terms of the changes in the material microstructure.

(2) The process of annealing can be divided into three stages, corresponding to the temperature ranges of $293-450 \mathrm{~K}, 450-525 \mathrm{~K}$, and $525-700 \mathrm{~K}$. The first stage is characterised by a relatively constant grain size and resistivity, virtually complete relaxation of internal stresses, and smooth decrease in microhardness. The second stage is characterised by intense grain growth and abruptly decreasing microhardness and resistivity in the absence of internal stresses. At the third stage, the grains continue to grow, the microhardness decreases smoothly, and the resistivity remains virtually unaltered.

(3) It is shown that at the first stage, the annealing causes the redistribution of dislocations and defects in the grain-boundary regions. The second stage is characterised by an intense recrystallization causing an abrupt decrease in the densities of grain-boundary defects and dislocations. The third stage is associated with a more complete annealing of defects and intense grain growth.

(4) The nanocrystalline state can be considered to be stable at temperatures of up to $400 \mathrm{~K}$.

\section{Acknowledgments}

This work was supported by the State Committee for Scientific Research under grant No. PBZ-KBN-013/T08/05 and grant "Nanomaterials and Supermolecular Systems" RAS.

\section{References}

[1] H. Gleiter, Prog. Mater. Sci. 33, 223 (1989).

[2] C.C. Koch, Nanostruct. Mater. 2, 109 (1993).

[3] R.Z. Valiev, A.V. Korznikov, R.R. Mulyukov, Mater. Sci. Eng. A 168, 141 (1993).

[4] B.D. Gullity, Elements of X-ray Diffraction, Addison-Wesley, Reading Mass. 1969. 
[5] J. Languillame, P. Chmelik, G. Kapelsky, F. Bordeaux, A.A. Nazarov, G. Canova, C. Esling, R.Z. Valiev, B. Baudelet, Acta Metall. Mater. 41, 1033 (1993).

[6] N.D. Zemtsova, Phys. Met. Metallogr. 77, 443 (1994).

[7] V.A. Likhachev, M.M. Myshlyaev, O.V. Sen'kov, Zakonomernosti sverkhplasticheskogo techeniya alyuminiyu pri kruchenii (Superplastic Behavior of Aluminum upon Torsion), Inst. Fiz. Tverd. Tela Akad. Nauk SSSR, Chernogolovka 1981, p. 1.

[8] M.M. Myshlyaev, R.Z. Valiev, D.R. Chalaev, Metallofizika 3, 72 (1981).

[9] Yu.V. Ivanisenko, A.V. Korznikov, I.M. Safarov, M.M. Myshlyaev, R.Z. Valiev, Izv. Ros. Akad. Nauk, Met., no. 6, 126 (1995).

[10] A.A. Nazarov, A.E. Romanov, R.Z. Valiev, Nanostruct. Mater. 4, 93 (1994).

[11] R.Z. Valiev, R.Sh. Musalimov, Phys. Met. Metallogr. 78, 666 (1994).

[12] J. Friedel, Dislocations, Pergamon, Oxford 1964.

[13] P.B. Hirsch, A. Howie, R.B. Nicholson, Electron Microscopy of Thin Crystals, Butterworth, London 1965. 